\title{
Quality Assessment of Licorice Based on Quantitative Analysis of Multicomponents by Single Marker Combined with HPLC Fingerprint
}

\author{
Tingting Li, ${ }^{1,2}$ Hao Zhou, ${ }^{1,3}$ Jiahua Ma, ${ }^{1,3}$ Lin Dong, ${ }^{1,3}$ Fang Xu $\mathbb{D}^{1},{ }^{1}$ and Xueyan Fu $\mathbb{D}^{1,2,3}$ \\ ${ }^{1}$ Ningxia Medical University, Yinchuan, China \\ ${ }^{2}$ Key Laboratory of Hui Ethnic Medicine Modernization, Ministry of Education (Ningxia Medical University), Yinchuan, China \\ ${ }^{3}$ School of Pharmacy, Ningxia Medical University, Yinchuan, China
}

Correspondence should be addressed to Xueyan Fu; xueyanfu2661@163.com

Received 2 September 2020; Revised 23 October 2020; Accepted 18 January 2021; Published 31 January 2021

Academic Editor: Li Zhang

Copyright (c) 2021 Tingting Li et al. This is an open access article distributed under the Creative Commons Attribution License, which permits unrestricted use, distribution, and reproduction in any medium, provided the original work is properly cited.

Licorice is a commonly used traditional Chinese medicine and natural sweetening agent, rich in numerous bioactive compounds. Moreover, it is one of the oldest and most frequently employed folk medicines in both eastern and western countries. It is prescribed for the treatment of asthma, fever, and cough. However, with the increasing demand of licorice, its quality and safety become the important issue. The content in licorice varies significantly in materials from different geographical origins. In this study, a reasonable and feasible evaluation method for the quality assessment of licorice was developed based on the analysis of high-performance liquid chromatography (HPLC) fingerprint, combined with the quantitative analysis of multicomponents by single marker (QAMS) method. Glycyrrhizic acid was selected as the internal reference substance, and ten components were simultaneously determined based on relative correction factors. The contents of eleven components in 21 batches of licorice were determined by the QAMS and the ESM (external standard method); there was no significant difference by comparison of the quantitative results between the QAMS and the ESM method; the cosine value $\left(C_{i r}>0.9999\right)$ confirmed the consistency of the two methods. According to the outcomes of 21 batches of licorice samples, the contents of the eleven components were used for further chemometric analysis. All of the samples of licorice from various geographical origins were divided into five categories based on hierarchical cluster analysis, which indicated the crucial influence of geographical origins on licorice. This study showed that QAMS combined with HPLC fingerprint and chemometrics methods could effectively control the quality of licorice. Hence, QAMS is a feasible and promising method for promoting the quality control standardization process of herbal medicines.

\section{Introduction}

Licorice is the dried root and rhizome of Glycyrrhiza uralensis Fisch., Glycyrrhiza inflata Bat., and Glycyrrhiza glabra L., used as an herbal medicine and natural sweetener in ancient Egyptian, Greece, and China [1,2]. It is a "necessary herbal medicine" in traditional Chinese medicine (TCM) and almost constitutes about $60 \%$ of all TCM prescriptions [3]. This botanical drug has been extensively used to treat the allergic-inflammatory disease, gastrointestinal problems, cardiovascular disease, cancer, and bladder and kidney ailments [4]. Modern pharmacological studies verified that it has the efficacy of the anti-inflammatory, anticancer, antibacterial, antispasmodic, antiallergic, antioxidative, antiAIDS, and so on [5].

At present, more than 400 compounds have been isolated and identified from licorice. The flavones and saponins are the main representative components in licorice. With the continuous expansion of the licorice market, the quality control of licorice is of increasing importance. Only glycyrrhizic acid is used for the indicators to assess the quality of licorice in European Pharmacopoeia (EP 9.0) [6], United States Pharmacopeia (USP 42) [7], and Japanese Pharmacopoeia (JP 17) [8]. Other important ingredients have been ignored, resulting in insufficient information on the integral chemical composition of licorice. In addition, Chinese 
Pharmacopeia (2020 version) records that liquiritin (content should not be less than $0.50 \%$ ) and glycyrrhizic acid (content should not be less than $2.00 \%$ ) were selected as quality control indicators [9]. However, each Chinese herbal medicine is a comprehensive complex with multiplicity of active components, contributing to pharmacological effects through synergy and mutual effect based on the theory of TCM [10]. Therefore, it is inadequate to use a few components to control the quality of complex plant products and traditional Chinese medicine. According to phytochemical studies, liquiritin, isoliquiritin apioside, isoliquiritin, neoisoliquiritin, isoliquiritigenin, formononetin, licochalcone $\mathrm{C}$, licochalcone $\mathrm{A}$, glabridin, and $18 \beta$-glycyrrhetinic acid widely exist in licorice [11]. And their pharmacological effects are significant, which are closely related to the efficacy of licorice. For example, liquiritin and glycyrrhizic acid could treat the inflammationrelated disorders [12]; neoisoliquiritin and formononetin inhibit inflammatory cytokines generation and improve antioxidant activity $[13,14]$; isoliquiritigenin, licochalcone $\mathrm{C}$, licochalcone $\mathrm{A}$, and glabridin have the efficacy of anticancer $[15,16]$; isoliquiritin has therapeutic efficacy on neuropathic hypersensitivity [2]. Therefore, selecting these 11 components as new quality control indicators can comprehensively characterize the chemical properties, medicinal functions, and intrinsic quality of licorice.

In many studies, the ESM has been used to analyze the concentrations of multiple components. However, this method has higher requirements of reference standard which requires more time and high experimental cost to isolation and purification [17]. As an alternative method, quantitative analysis of multicomponents by single marker (QAMS) only needs a single reference standard to simultaneously analyze the contents of multicomponents, which is more available and efficient for the quality control (QC) [18]. This method could reduce not only the cost but also the detection time of experiment [19]. Additionally, QAMS has been widely used in QC to effectively ensure the safety of herbal or botanical products effectively. And it has gradually been recognized by researchers and successfully employed for the analysis of medicinal herb [20]. Since this method was firstly proposed by Dr. Wang in China, it has received more attention and acceptance in the world. And in American Herbal Pharmacopoeia, it is even applied for the quantification study of 20 variants of plants [21].

In recent years, the chromatographic fingerprint analysis has also quite been recommended by academia community as a useful method for the quality control of medicinal plants and herbal products. Furthermore, this method has been accepted as a strategy for the quality assessment of herbal medicines and preparations by the US Food and Drug Administration, State Food and Drug Administration of China, and the European Medicines Agency [22]. Fingerprint technology emphasizes overall characterization, in order to distinguish some substitutes and adulterants from the real original medicine by chromatographic fingerprinting based on the presence or absence of a limited number of peaks. Therefore, we combine characteristic fingerprints with QAMS, which can more effectively and comprehensively improve the quality evaluation of licorice.
In this study, we established a comprehensive and effective quality evaluation method for licorice, which integrated HPLC fingerprint characterization technology, combined with the QAMS method, and chemometric analysis. This strategy not only provides comprehensive chemical information but also can quickly and reliably determine various components and evaluate quality consistency. This innovative method could be beneficial for simplifying the processes in the quality control of licorice and promoting the quality control standardization process of herbal medicines.

\section{Materials and Methods}

2.1. Plant Materials. 21 batches of dried licorice (Table 1 and Figure 1) were collected from different areas (including Ningxia, Gansu, Inner Mongolia, Xinjiang, and Heilongjiang) of China and identified by Prof. Zhang Xinhui, Ningxia Medical University, Yinchuan, China.

2.2. Reagents and Chemicals. Acetonitrile (purity 100\%) of HPLC grade was from Thermo Fisher Scientific Company. Glacial acetic acid (purity 100\%, HPLC grade) was acquired from Kaixin Chemical Industry Co. Ltd. (Tianjin, China). Methanol and ethanol were analytical grade. These reference substances of liquiritin (L1), isoliquiritin apioside (L2), isoliquiritin (L3), neoisoliquiritin (L4), isoliquiritigenin (L5), formononetin (L6), licochalcone C (L7), licochalcone A (L8), glabridin (L9), glycyrrhizic acid (L10), and 18 $\beta$ glycyrrhetinic acid (L11) were purchased from Yuanye Biotechnology Co., Ltd. (Shanghai, China). The purity of each compound was verified to be greater than $98 \%$ by highperformance liquid chromatography (HPLC) using the peak area normalization method. The chemical structures of these compounds are shown in Figure 2. All other chemicals used were of analytical grade.

2.3. Preparation of the Sample Solution. Powder sample of licorice was accurately weighed $(0.2 \mathrm{~g})$ and added in $25 \mathrm{~mL}$ of $70 \%$ ethanol in $50 \mathrm{~mL}$ triangular flask, sonicated for $30 \mathrm{~min}$, and allowed to cool to room temperature. Additional $70 \%$ ethanol was complemented for weightlessness. All of the sample solutions were filtered through a $0.22 \mu \mathrm{m}$ Millipore filter prior to HPLC analysis.

2.4. Preparation of the Standard Solution. The reference solution of licorice was prepared by accurately dissolving weighed samples of each compound in $70 \%$ methanol, making a mixture of $43.85 \mu \mathrm{g} / \mathrm{mL}$ of liquiritin, $40.77 \mu \mathrm{g} / \mathrm{mL}$ of isoliquiritin, $36.92 \mu \mathrm{g} / \mathrm{mL}$ of neoisoliquiritin, $40 \mu \mathrm{g} / \mathrm{mL}$ of isoliquiritin apioside, $41.54 \mu \mathrm{g} / \mathrm{mL}$ of isoliquiritigenin, $30.77 \mu \mathrm{g} / \mathrm{mL}$ of formononetin, $30.07 \mu \mathrm{g} / \mathrm{mL}$ of glabridin, $33.07 \mu \mathrm{g} / \mathrm{mL}$ of licochalcone $\mathrm{A}, 30.77 \mu \mathrm{g} / \mathrm{mL}$ of licochalcone $\mathrm{C}, 185 \mu \mathrm{g} / \mathrm{mL}$ of $18 \beta$-glycyrrhetinic acid, and $240 \mu \mathrm{g} / \mathrm{mL}$ of glycyrrhizic acid, mixed evenly. All the standard solutions were stored in a refrigerator at $4^{\circ} \mathrm{C}$, before use. 
TABle 1: The information of licorice from different producing areas.

\begin{tabular}{lc}
\hline No. & Producing areas \\
\hline S1 & Wuzhong, Ningxia, China \\
S2 & Wuzhong, Ningxia, China \\
S3 & Wuzhong, Ningxia, China \\
S4 & Wuzhong, Ningxia, China \\
S5 & Guyuan, Ningxia, China \\
S6 & Guyuan, Ningxia, China \\
S7 & Lanzhou, Gansu, China \\
S8 & Lanzhou, Gansu, China \\
S9 & Wuwei, Gansu, China \\
S10 & Dingxi, Gansu, China \\
S11 & Dingxi, Gansu, China \\
S12 & Qingyang, Gansu, China \\
S13 & Baiyin, Gansu, China \\
S14 & Chifeng, Inner Mongolia, China \\
S15 & Chifeng, Inner Mongolia, China \\
S16 & Ordos, Inner Mongolia, China \\
S17 & Lvliang, Shanxi, China \\
S18 & Alaer, Xinjiang, China \\
S19 & Ili Kazak Autonomous Prefecture, Xinjiang, China \\
S20 & Ili Kazak Autonomous Prefecture, Xinjiang, China \\
S21 & Harbin, Heilongjiang, China \\
\hline
\end{tabular}

2.5. Chromatographic Procedures. Chromatographic analysis was performed by using an Agilent 1260 series system (Agilent Technologies, MA, USA) equipped with an online degassing system, an autosampler, and a thermostated column compartment. Agilent ZORBAX SB-C18 Column $(4.6 \times 250 \mathrm{~mm}, 5 \mu \mathrm{m})$ (Agilent Technologies, USA) was used for chromatographic separation of target compounds. The mobile phase was made up of $0.2 \%$ glacial acetic acid (A) and acetonitrile (B) with the following optimized gradient elution process: $0 \sim 15 \mathrm{~min}, 15 \% \mathrm{~B} ; 15 \sim 25 \mathrm{~min}, \quad 15 \sim 20 \% \mathrm{~B}$; 25 70 min, $20 \sim 50 \% \mathrm{~B} ; 70 \sim 90 \mathrm{~min}, 50 \sim 70 \% \mathrm{~B} ; 90 \sim 95 \mathrm{~min}$, $70 \sim 15 \% \mathrm{~B}$. The detection wavelength was $254 \mathrm{~nm}$. The column temperature was set at $30^{\circ} \mathrm{C}$. The flow velocity was $1.0 \mathrm{~mL} / \mathrm{min}$ with a sample injection volume of $20 \mu \mathrm{L}$.

2.6. Method Validation. Eleven reference compounds were accurately weighted and separately dissolved in ethanol to obtain appropriate concentrations. The stock solutions were serially diluted with ethanol to a certain concentration range to obtain a calibration curve. The linear calibration curves were constructed by six different concentrations of mixed standard solutions. The limits of detection (LODs) and limits of quantification (LOQs) were determined at signal-to-noise ratio of 3 and 10, respectively. The evaluation of precision was examined from six-time analysis of the licorice sample placed in the autosampler. And the stability test was conducted by a six-time analysis of the same licorice sample at different time points $(0,3,6,9,12$, 18 , and $24 \mathrm{~h}$ ) after solution preparation at room temperature $\left(25 \pm 5^{\circ} \mathrm{C}\right)$. The repeatability was evaluated according to the assay of six duplicates. Relative standard deviations (RSDs) of relative peak areas (RPAs) of all the analytes were used for evaluation. As to the accuracy investigation, a recovery test was conducted with conventional standard addition method at different concentration following equation (1):

$$
\operatorname{recovery}(\%)=\frac{(\text { detected amount }- \text { original amount })}{\text { added amount }} \times 100 \% \text {. }
$$

\subsection{QAMS Quality Evaluation Model for Licorice}

2.7.1. RCF Calculation and Its Tolerance Investigation. The calculation methods of relative correction factor (RCF) have been previously reported. Firstly, glycyrrhizic acid is used as the internal standard, and a multipoint method (equation (2)) was used to calculate the relative correction factor of liquiritin, isoliquiritin, neoisoliquiritin, isoliquiritin apioside, isoliquiritigenin, formononetin, glabridin, licochalcone $\mathrm{A}$, licochalcone $\mathrm{C}$, and $18 \beta$-glycyrrhetinic acid. The reproducibility of the correction factors was investigated at different column, using the different instruments. The relative correction factor is calculated as

$$
f_{s i}=\frac{f_{s}}{f_{i}}=\frac{\left(A_{s} / C_{s}\right)}{\left(A_{i} / C_{i}\right)}
$$

where $A_{s}$ is the peak area of the internal reference substance, $A_{i}$ is the peak area of sample $i, C_{s}$ is the concentration of the internal reference substance, and $C_{i}$ is the concentration of sample $i$.

2.7.2. Assessment of the ESM and the QAMS Method. In order to verify the feasibility of QAMS, the validated traditional ESM and QAMS methods were employed to evaluate the similarity of the 21 batches of licorice samples from different producing regions. For ESM, the content of the eleven components was determined by eleven reference standards (L1-L11), whereas for QAMS, the results were based on the properties of the calculation of RCF, the content of the selected internal substance (L10) was determined to be similar to ESM, and then the contents of the other ten components were calculated based on the RCF between analytes and the internal substance [23]. In this study, the contents of liquiritin, isoliquiritin, neoisoliquiritin, isoliquiritin apioside, isoliquiritigenin, formononetin, glabridin, licochalcone $\mathrm{A}$, licochalcone $\mathrm{C}$, and $18 \beta$-glycyrrhetinic acid were calculated using the external standard method and QAMS, and glycyrrhizic acid as the internal standard. The content of the multimarker components measured by QAMS was compared with results from ESM, using the correlation coefficient method $(r)$ (equation (3)) and the cosine ratio value $\left(C_{i r}\right)$ (Equation (4)) method to verify the feasibility of QAMS [24, 25]. The angle cosine value $\left(C_{i r}\right)$ and the correlation coefficient $(r)$ are commonly used to evaluate the similarity of TCM. A larger $C_{i r}$ value indicates a higher similarity of the target samples [26]. Meanwhile, the correlation coefficient from 0 (no correlation) to 1 (full correlation) reflects the closeness between the two variables [27]. $C_{i r}$ and $r$ were calculated by using Microsoft Excel 2019 software in this study. 


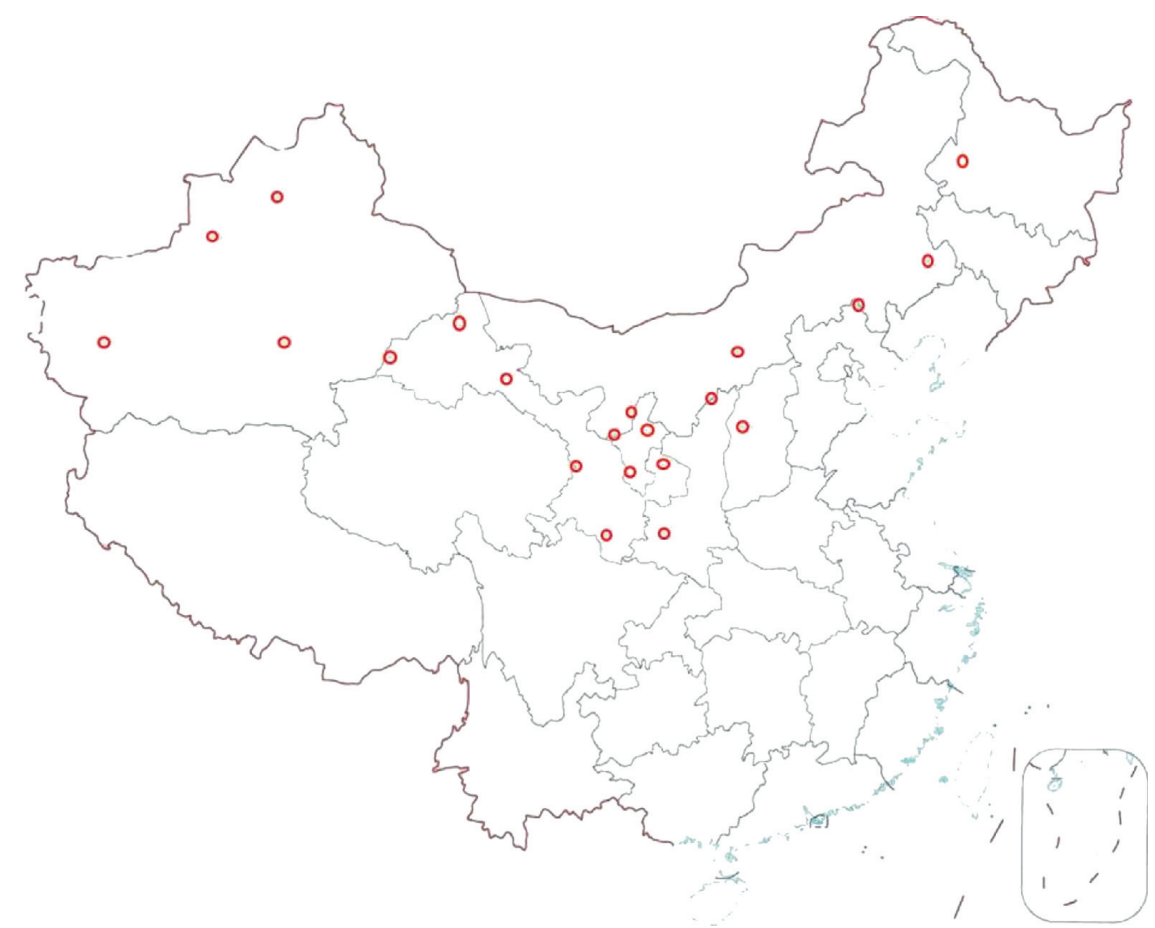

FIGURE 1: Licorice sampling locations in different regions in China.

$$
\begin{aligned}
r & =\frac{\sum_{i=1}^{n}\left(X_{i}-\bar{X}\right)\left(Y_{i}-\bar{Y}\right)}{\sqrt{\sum_{i=1}^{n}\left(X_{i}-\bar{X}\right) \sqrt{\sum_{i=1}^{n}\left(Y_{i}-\bar{Y}\right)}}} \\
C_{i r} & =\frac{\sum_{k=1}^{n} X_{i k} \cdot X_{r k}}{\sqrt{\left(\sum_{k=1}^{n} X_{i k}^{2}\right)\left(\sum_{k=1}^{n} X_{r k}^{2}\right)}}
\end{aligned}
$$

where $X_{i k}$ is the value of compound $k$ in sample $i$ in the ESM and $X_{r k}$ is the value of compound $k$ of the same sample in the QAMS method.

2.8. Data Analysis. The data were analyzed and assessed by the specialized software, named Similarity Evaluation System for Chromatographic Fingerprint of Traditional Chinese Medicine (TCM) (Version 2012.130723, Chinese Pharmacopoeia Committee). The similarity among different chromatograms was calculated by the correlative coefficient. HCA is a chemical pattern recognition and classification evaluation method, which is used to classify objects (samples) into clusters, such that each object is similar to the others within a cluster, but different from those in other clusters with respect to a predetermined selection criterion [28]. Using the peak areas of the eleven compounds, quality variations of the 21 batches of licorice samples were evaluated by the HCA with the software of SPSS (International Business Machines Corporation, New York, NY, USA) 22.0 to further investigate the difference among the licorice samples.

\section{Results}

3.1. The Optimization of the Chromatographic Separation Method. It is important to select a suitable extraction method and HPLC parameters for effective chromatograms with good separation. Extraction solvent (70\% ethanol, 70\% methanol, and water), extraction method (ultrasonic extraction, heated reflux extraction, and decocting method), extraction time $(30,60$, and $90 \mathrm{~min})$, and extraction times (once, twice, and three times) were investigated for the higher extraction efficiency. The specific steps were eventually demonstrated to be the most suitable as the section of preparation of sample preparation described.

Several mobile phase systems including methanol-water, acetonitrile-water, methanol with $0.2 \%$ glacial acetic acid, and acetonitrile with $0.2 \%$ glacial acetic acid were tested to optimize the chromatographic conditions. Flow velocity $\left(0.8,1\right.$, and $\left.1.2 \mathrm{~mL} \cdot \mathrm{min}^{-1}\right)$ was examined and compared. An optimized HPLC condition was determined with a thorough comparison on the resolution, base line, elution time, and number of characteristic peaks.

\subsection{HPLC Method Validation}

3.2.1. Linearity, $L O D$, and $L O Q$. The calibration curves of the eleven standard substances were plotted with a series of mixed standard solutions with gradient concentration. The linearity of each component was evaluated with different concentrations and the corresponding peak areas by plotting its calibration curve. Then, standard solution of the individual component was diluted gradually; the limit of quantification (LOQ) and limit of detection (LOD) values were determined by using signal-to-noise ratios of $3: 1$ and $10: 1$, respectively. As shown in Table 2, the high correlation coefficient values $\left(R^{2}>0.9990\right)$ show a good linear relationship over a wide concentration range, and the calibration curve could be used for quantitative analysis within a given concentration range. 
<smiles>O=C1C[C@H](c2ccc(O[C@@H]3O[C@H](CO)[C@@H](O)[C@H](O)[C@H]3O)cc2)Oc2cc(O)ccc21</smiles>

L1<smiles>O=C1/C(=C/c2ccc(O[C@@H]3O[C@H](CO)[C@@H](O)[C@H](O)[C@H]3O)cc2)COc2cc(O)ccc21</smiles>

L3<smiles>O=C(/C=C/c1ccc(O)cc1)c1ccc(O)cc1O</smiles>

L5

$\mathrm{HO}$<smiles>COc1c(/C=C2\Oc3cc(O)ccc3C2=O)ccc(O)c1CC=C(C)C</smiles>

L7

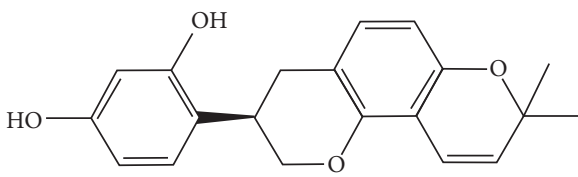

L9

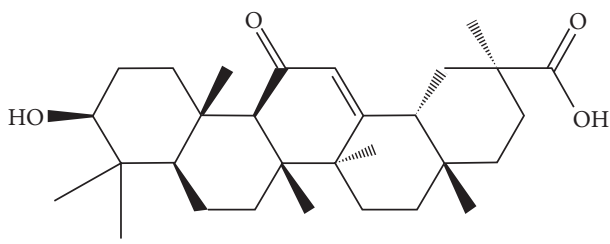

L11<smiles>O=C(/C=C/c1ccc(O[C@@H]2O[C@H](CO)[C@@H](O)[C@H](O)[C@H]2O[C@@H]2OC[C@@](O)(CO)[C@H]2O)cc1)c1ccc(O)cc1O</smiles><smiles>O=C(/C=C/c1ccc(O)cc1)c1ccc(O[C@@H]2O[C@H](CO)[C@@H](O)[C@H](O)[C@H]2O)cc1O</smiles>

L4

$\mathrm{HO}$<smiles>COc1ccc(-c2coc3cc(C)ccc3c2=O)cc1</smiles>

L6<smiles>C=CC(C)(C)c1cc(/C=C/C(=O)c2ccc(O)cc2)c(OC)cc1O</smiles>

L8

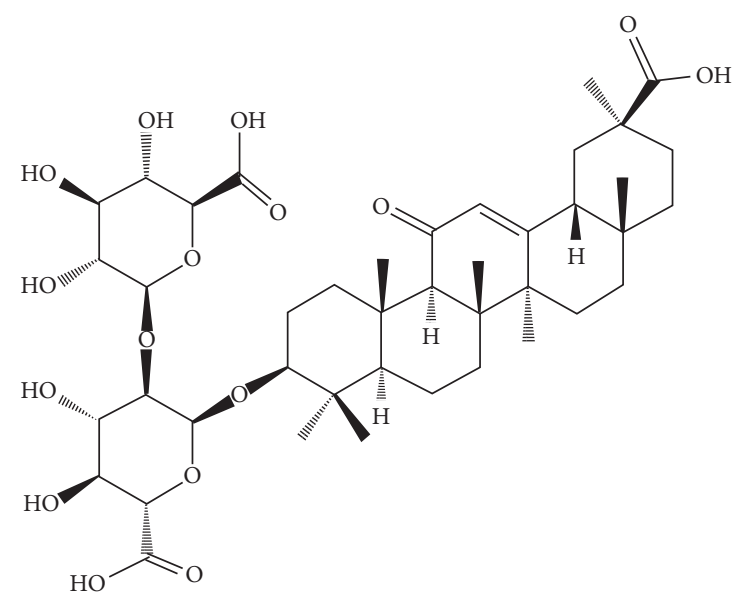

L10

Figure 2: Chemical structures of the 11 target compounds. 
TABle 2: The regression equations, limits of detections (LODs), and limits of quantity (LOQs) of eleven compounds.

\begin{tabular}{lccccc}
\hline Analytes & Regression equation & $R$ & Linear ranges $(\mu \mathrm{g} / \mathrm{mL})$ & LOD $(\mu \mathrm{g} / \mathrm{mL})$ & LOQ $(\mu \mathrm{g} / \mathrm{mL})$ \\
\hline L1 & $Y=632,698 X+12922$ & 0.9999 & $0.006 \sim 10.600$ & 0.056 & 0.020 \\
L2 & $Y=671,520 X+167.53$ & 0.9999 & $0.006 \sim 10.000$ & 0.002 & 0.047 \\
L3 & $Y=771,628 X-17609$ & 0.9999 & $0.136 \sim 3.533$ & 0.058 & 0.066 \\
L4 & $Y=1,715,420.24 X-3,985.90$ & 0.9999 & $0.004 \sim 0.615$ & 0.041 & 0.037 \\
L5 & $Y=1,631,646.21 X+7,366.99$ & 0.9999 & $0.006 \sim 0.831$ & 0.032 & 0.186 \\
L6 & $Y=2,861,225.81 X-4,336.31$ & 0.9999 & $0.011 \sim 0.738$ & 0.012 & 0.113 \\
L7 & $Y=2,401,806.09 X-3,173.10$ & 0.9999 & $0.005 \sim 0.615$ & 0.048 & 0.097 \\
L8 & $Y=1,837,868.82 X-9,926.36$ & 0.9999 & $0.005 \sim 9.200$ & 0.038 \\
L9 & $Y=798,408 X-494.05$ & 0.9999 & $0.002 \sim 0.661$ & 0.044 & 0.157 \\
L10 & $Y=762,911 X-21434$ & 0.9999 & $0.400 \sim 14.000$ & $0.007 \sim 0.800$ & 0.135 \\
L11 & $Y=1,496,816.51 X-7,318.86$ & 0.9999 & & & 0.03 \\
\hline
\end{tabular}

TABLE 3: The precision, stability, repeatability, and recovery of the analytical method.

\begin{tabular}{|c|c|c|c|c|c|c|c|c|}
\hline \multirow{3}{*}{ Analytes } & \multirow{2}{*}{\multicolumn{2}{|c|}{$\begin{array}{c}\text { Stability } \\
\text { RSD }(\%, n=6)\end{array}$}} & \multirow{2}{*}{\multicolumn{2}{|c|}{$\begin{array}{c}\text { Precision } \\
\text { RSD }(\%, n=6)\end{array}$}} & \multirow{2}{*}{\multicolumn{2}{|c|}{$\begin{array}{c}\text { Repeatability } \\
\text { RSD }(\%, n=6)\end{array}$}} & \multicolumn{2}{|c|}{ Recovery } \\
\hline & & & & & & & \multirow{2}{*}{ Mean (\%) } & \multirow{2}{*}{ RSD (\%) } \\
\hline & RRT & RPA & RRT & RPA & RRT & RPA & & \\
\hline L1 & 0.72 & 0.29 & 0.31 & 0.30 & 0.62 & 0.28 & 93.8 & 1.47 \\
\hline $\mathrm{L} 2$ & 0.21 & 0.22 & 0.11 & 0.27 & 0.22 & 1.66 & 92.5 & 1.94 \\
\hline L3 & 0.18 & 0.28 & 0.09 & 0.34 & 0.20 & 0.29 & 103.5 & 2.02 \\
\hline L4 & 0.16 & 1.68 & 0.07 & 2.34 & 0.20 & 2.97 & 108.4 & 2.89 \\
\hline L5 & 0.16 & 1.53 & 0.06 & 1.83 & 0.12 & 1.81 & 86.5 & 2.58 \\
\hline L6 & 0.12 & 1.37 & 0.08 & 0.65 & 0.10 & 1.52 & 94.6 & 1.38 \\
\hline L7 & 0.10 & 0.44 & 0.07 & 0.57 & 0.10 & 0.56 & 98.1 & 2.34 \\
\hline L8 & 0.10 & 1.55 & 0.07 & 0.68 & 0.10 & 1.64 & 102.7 & 2.86 \\
\hline L9 & 0.08 & 0.61 & 0.05 & 0.49 & 0.08 & 0.18 & 89.5 & 2.81 \\
\hline L10 & 0.12 & 0.74 & 0.09 & 0.59 & 0.10 & 0.87 & 105.8 & 2.59 \\
\hline L11 & 0.08 & 2.52 & 0.25 & 2.69 & 0.65 & 2.68 & 92.07 & 1.08 \\
\hline
\end{tabular}

3.2.2. Stability, Precision, Repeatability, and Accuracy. The RSD values for the precision are summarized in Table 3. From the results, the RSD results of eleven components for the instrument precision were in the range of $0.27 \%-2.69 \%$, which were all lower than $3 \%$. This indicates that this method has a good precision.

The peak areas of L1-L11 were recorded, and the values of RSD were $0.29 \%, 0.22 \%, 0.28 \%, 1.68 \%, 1.53 \%, 1.37 \%$, $0.44 \%, 1.55 \%, 0.61 \%, 0.74 \%$, and $2.52 \%$, respectively. Therefore, the sample solution was stable at room temperature within $24 \mathrm{~h}$ (Table 3 ).

There was good accuracy because the average recovery rates of eleven compounds varied in the range of $86.5 \%-$ $102.7 \%$. Meanwhile, RSD values of recovery rates for each compound were lower than $3 \%$ totally.

3.3. Chromatographic Fingerprint Analysis and Similarity. The fingerprint chromatograms of 21 batches of licorice samples were recorded as shown in Figure 3(a). A chromatogram of licorice sample (Figure 3(c)) was developed with the mean of all the chromatograms by the Similarity Evaluation System for Chromatographic Fingerprint of Traditional Chinese Medicine (version 2012). Based on comparison of the chromatograms of the mixed reference standard solution (Figure $3(\mathrm{~b})$ ), eleven common peaks (L1-L11) of fingerprint of licorice were identified, respectively, being liquiritin, isoliquiritin apioside, isoliquiritin, neoisoliquiritin, isoliquiritigenin, formononetin, licochalcone $\mathrm{C}$, licochalcone $\mathrm{A}$, glabridin, glycyrrhizic acid, and $18 \beta$-glycyrrhetinic acid. The peak value of L10 was selected as the reference peak with relative retention time and the ratio of peak area as shown in Figure 3(c). As a result, their similarity values calculated based on similarity system theory of 21 samples were $>0.617$, which meant that the common peaks were in good correlation. Therefore, glycyrrhizic acid was chosen as the internal standard to determine the contents of liquiritin, isoliquiritin, neoisoliquiritin, isoliquiritin apioside, isoliquiritigenin, formononetin, glabridin, licochalcone $\mathrm{A}$, licochalcone $\mathrm{C}$, and $18 \beta$-glycyrrhetinic acid.

\subsection{QAMS Method}

3.4.1. RCF Calculation and Its Robustness Investigation. The RCF for component I (internal standard) is the ratio of the absolute correction factor for component I to standard substance $S$ (reference compound). It is of great significance to select appropriate internal reference standards for the accurate determination of various components in TCM. Glycyrrhizic acid was used as an internal reference because it is inexpensive and easily obtained. And the values of RCF for the other ten compounds were computed in different concentrations according to equation (2). The average RCF of each compound is shown in Table 4. 


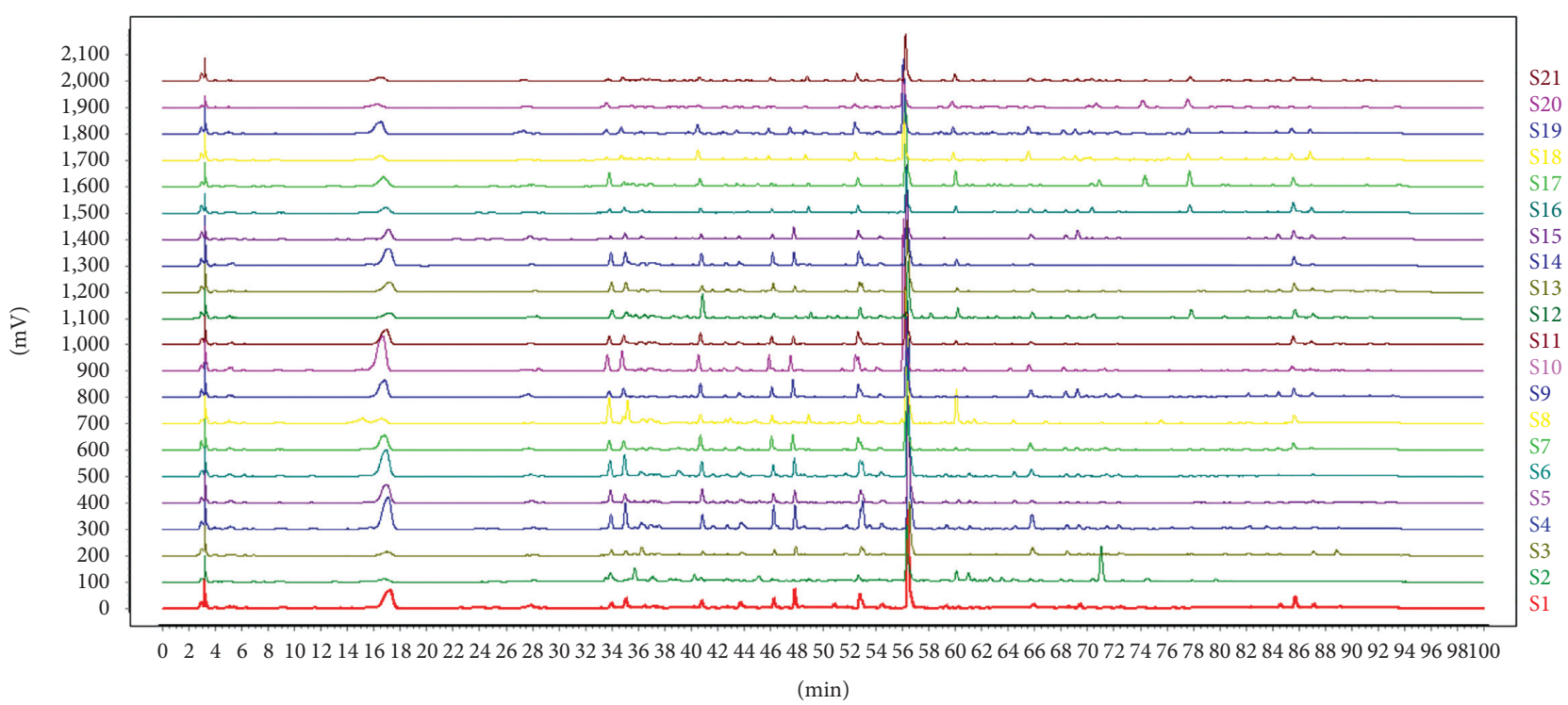

(a)

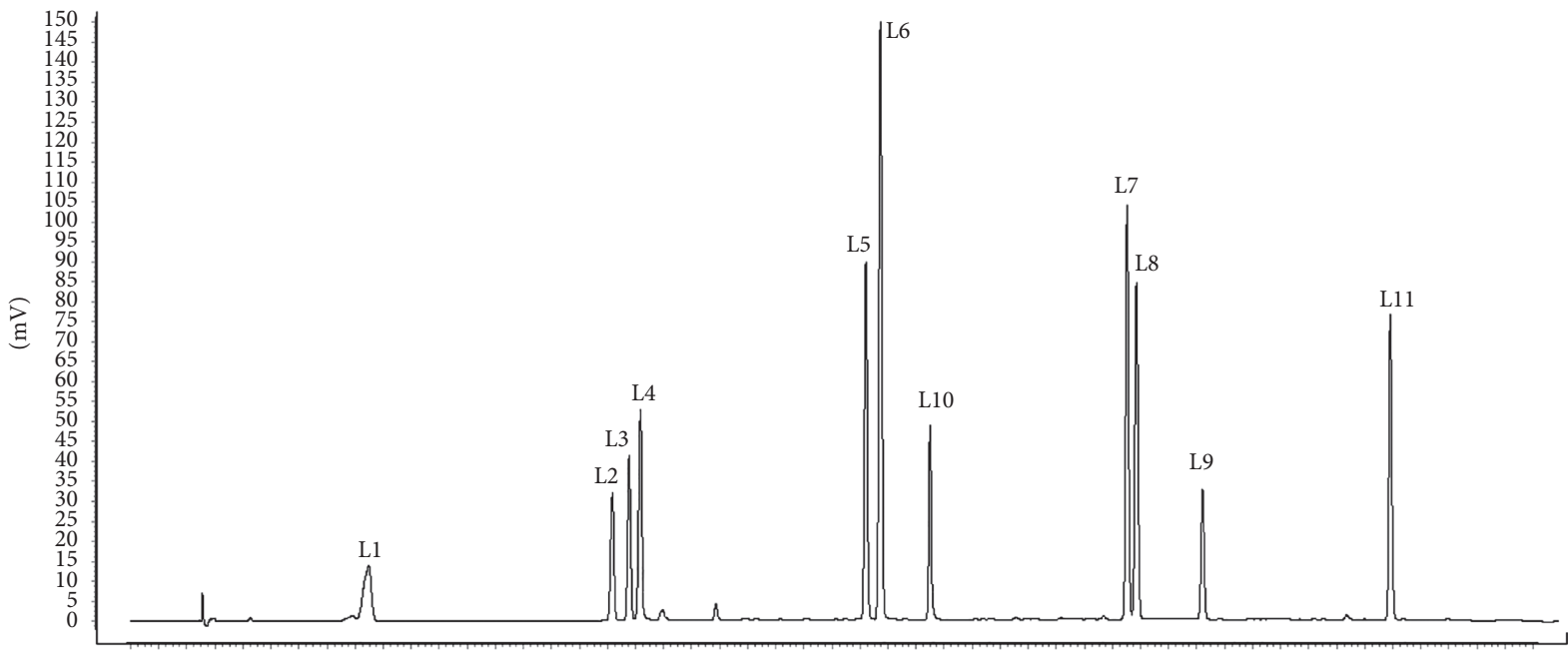

0244688101214161820222426283032343638404244464850525456586062646668707274767880828486889092949698100 (min)

(b)

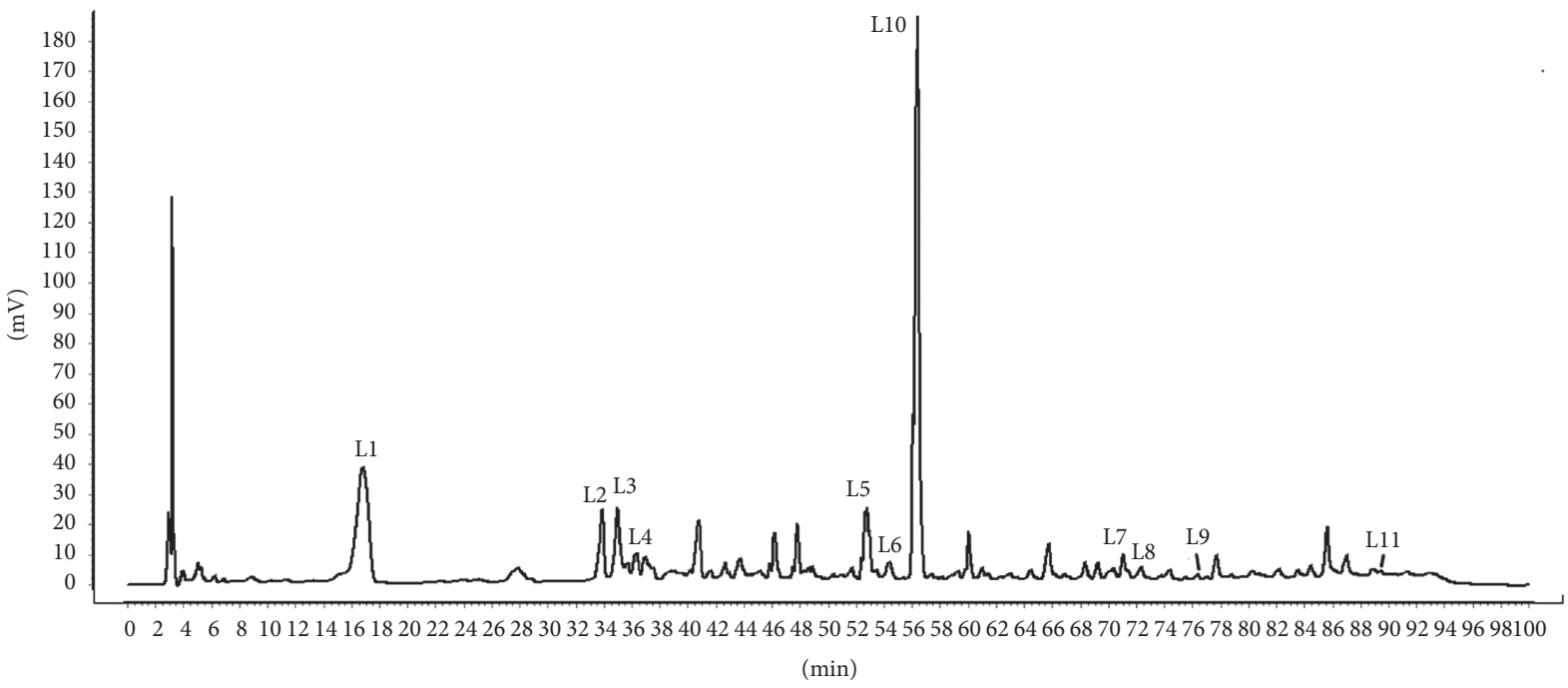

(c)

FiguRE 3: The HPLC fingerprints of the 21 batches of licorice (a); a chromatogram of the mixture of licorice standard compounds (b); a chromatogram of the licorice sample (c). 
TABLE 4: Results of RCF for the ten compounds.

\begin{tabular}{lcccccccc}
\hline Sample $(\mu \mathrm{l})$ & 1 & 2 & 5 & 7 & 10 & 15 & Average & RSD\% \\
\hline L1 & 0.8636 & 0.8703 & 0.8807 & 0.8916 & 0.8723 & 0.8457 & 0.8707 & 1.79 \\
L2 & 0.8752 & 0.8686 & 0.8659 & 0.8597 & 0.8513 & 0.8965 & 0.8695 \\
L3 & 1.0369 & 0.9736 & 0.9858 & 0.9751 & 0.9986 & 0.9703 & 0.9901 \\
L4 & 1.8007 & 1.7034 & 1.7939 & 1.6923 & 1.7906 & 1.7444 & 1.7542 \\
L5 & 1.9258 & 1.9433 & 1.9376 & 1.9955 & 1.9678 & 1.9682 & 1.9564 \\
L6 & 3.5247 & 3.2998 & 3.4185 & 3.4982 & 3.4655 & 3.3458 & 3.4254 \\
L7 & 3.1055 & 3.1986 & 3.2897 & 3.0687 & 3.2648 & 3.1359 & 3.1772 \\
L8 & 2.1988 & 2.2061 & 2.2993 & 2.2521 & 2.1687 & 2.1659 & 2.2152 \\
L9 & 0.9887 & 0.9586 & 0.9633 & 0.9986 & 0.9455 & 0.9854 & 0.9734 \\
L11 & 1.9963 & 1.9300 & 1.9770 & 1.9243 & 1.9685 & 1.9647 & 2.53 \\
\hline
\end{tabular}

TABLE 5: Results of robustness test for RCF.

\begin{tabular}{|c|c|c|c|c|c|c|c|c|c|c|c|}
\hline Instrument & Column $^{1}$ & L1 & $\mathrm{L} 2$ & L3 & $\mathrm{L} 4$ & L5 & L6 & L7 & L8 & L9 & L11 \\
\hline \multirow{3}{*}{ Agilent $1290+6545$} & $1 \#$ & 0.8457 & 0.8965 & 0.9703 & 1.7444 & 1.9682 & 3.4258 & 3.1359 & 2.1659 & 0.9854 & 1.9647 \\
\hline & $2 \#$ & 0.8679 & 0.8806 & 0.9651 & 1.7515 & 1.9721 & 3.4312 & 3.1583 & 2.1789 & 1.0416 & 1.9832 \\
\hline & 3\# & 0.8564 & 0.8717 & 0.9657 & 1.7555 & 1.9628 & 3.4451 & 3.0085 & 2.1539 & 1.0336 & 1.9988 \\
\hline \multirow{3}{*}{ Agilent 1260} & $1 \#$ & 0.8462 & 0.8959 & 0.9700 & 1.7450 & 1.9677 & 3.4266 & 3.1355 & 2.1652 & 0.9887 & 1.9651 \\
\hline & $2 \#$ & 0.8636 & 0.8698 & 0.9634 & 1.7556 & 1.9712 & 3.4296 & 3.1390 & 2.1720 & 1.0312 & 1.9765 \\
\hline & $3 \#$ & 0.8721 & 0.8497 & 0.9875 & 1.7572 & 1.9914 & 3.4184 & 3.1716 & 2.1963 & 1.0304 & 1.9963 \\
\hline \multirow{3}{*}{ Waters Alliance E2695 } & $1 \#$ & 0.8455 & 0.8962 & 0.9698 & 1.7467 & 1.9698 & 3.4254 & 3.1363 & 2.1677 & 0.9820 & 1.9691 \\
\hline & $2 \#$ & 0.8624 & 0.8834 & 0.9581 & 1.7390 & 1.9619 & 3.4259 & 3.1361 & 2.1673 & 1.0391 & 1.9752 \\
\hline & $3 \#$ & 0.8659 & 0.8419 & 0.9853 & 1.7498 & 1.9845 & 3.4316 & 3.1617 & 2.1899 & 1.0297 & 1.9893 \\
\hline Mean & & 0.8584 & 0.8762 & 0.9706 & 1.7494 & 1.9722 & 3.4288 & 3.1314 & 2.1730 & 1.0180 & 1.9798 \\
\hline RSD\% & & 1.21 & 2.29 & 1.01 & 0.35 & 0.49 & 0.21 & 1.54 & 0.61 & 2.44 & 0.65 \\
\hline
\end{tabular}

${ }^{1} 1 \#$ : DiKMA-C 18 ; 2\#: Agilent- $\mathrm{C}_{18} ; 3 \#$ : Thermo- $\mathrm{C}_{18}$.

In previous studies of QAMS, researchers have investigated the robustness of different columns, instruments, and flow rates [29]. In this study, the experiments on different instruments were carried out in different labs, using Agilent 1100, Agilent 1260, and Waters Alliance E2695 instruments. DiKMA-C 18 $(4.6 \mathrm{~mm} \times 250 \mathrm{~mm}, 5 \mu \mathrm{m})$, Agilent- $\mathrm{C}_{18}(4.6 \mathrm{~mm} \times 250 \mathrm{~mm}$, $5 \mu \mathrm{m})$, and Thermo- $\mathrm{C}_{18}(4.6 \mathrm{~mm} \times 250 \mathrm{~mm}, 5 \mu \mathrm{m})$ columns were used to study the effects of different columns. The results (Table 5) show that the RCF from different columns and instruments had good reproducibility with $\mathrm{RSD}<3 \%$.

3.4.2. Comparative Study of the QAMS and ESM Methods. In order to verify the availability of the QAMS method, the contents of eleven compounds in 21 batches of licorice were evaluated using the QAMS and ESM methods, respectively. The results revealed that $C_{i r} \geq 0.9998, r \geq 0.9936$ in Table 6, suggesting that no significant differences existed between the ESM and the QAMS method. Hence, the present QAMS method was reliable for the simultaneous quantification of the eleven components of the licorice.

3.5. Hierarchical Cluster Analysis. In order to quantitatively and objectively analyze licorice, the contents of eleven components in 21 batches of licorice samples were selected as a clustering variable to obtain a dendrogram. Euclidean distance is usually used to distinguish the similarity of samples; a larger Euclidean distance represents a greater difference and the similarity is relatively low [30]. From Figure 4, it can be seen that the 21 samples from different origins were separated into five clusters based on the dendrogram cut at a distance of 4.5. Group 1 is S5 from Ningxia in China; group 2 is S7 and S11 from Gansu in China; group 3 is S9 from Gansu in China; group 4 is S3, S4, S13, S16, S17, and S19; and group 5 contains the rest of the samples. Particularly, Ningxia has been considered as the Daodi production area (area which produces authentic and superior original medicine) of licorice in China. However, samples 1 to 6 from Ningxia showed different levels and ratios of chemical components. The possible explanation is that the licorice had been grown in different environments or in a different manner, resulting in the contents of the eleven components being different. The factors included soil type, climate, humidity, and temperature, harvesting time, planting patterns, dying methods, and other factors [31]. Therefore, the key of quality control of licorice is strictly controlling the different geographical sources to prevent adulteration.

\section{Discussion}

In this study, QAMS method was used to verify that the established method has high sensitivity and good precision, which is sufficient to simultaneously determine 11 


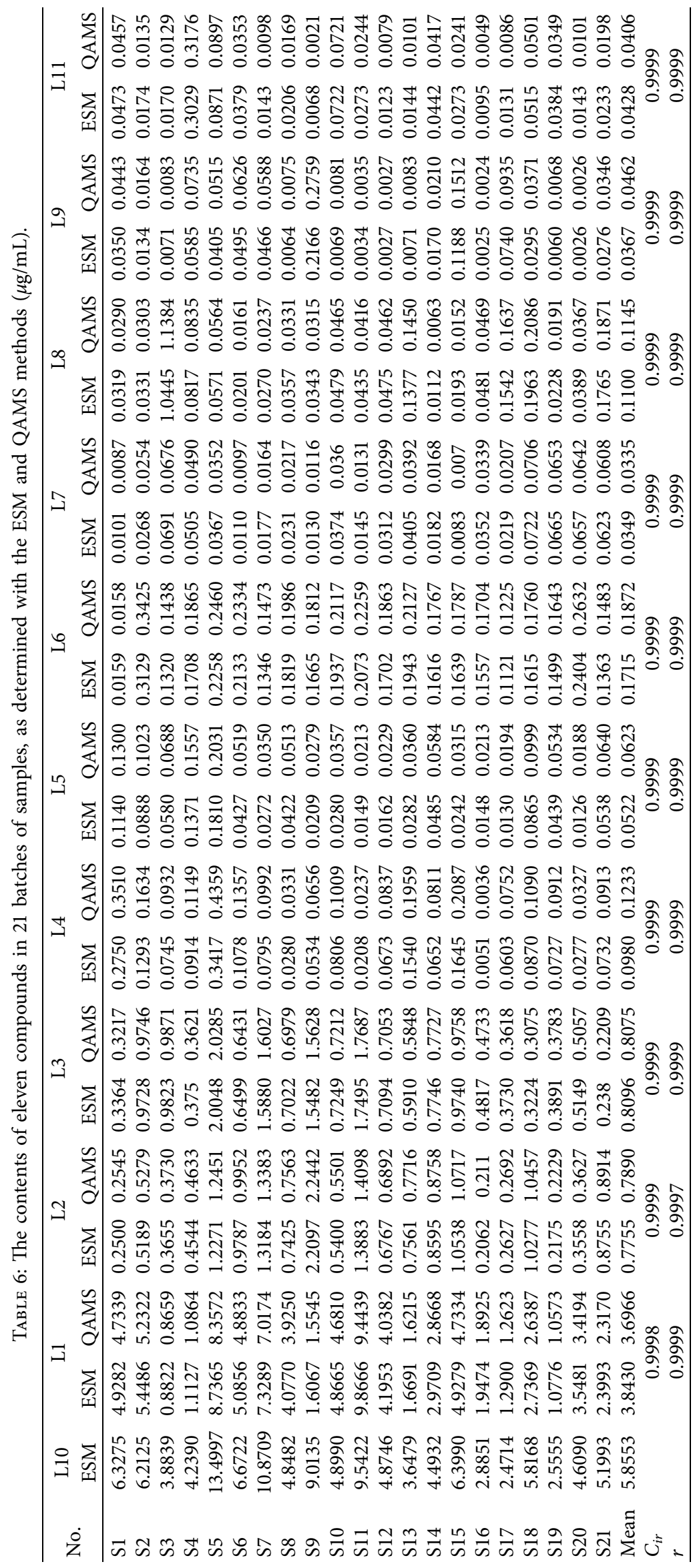




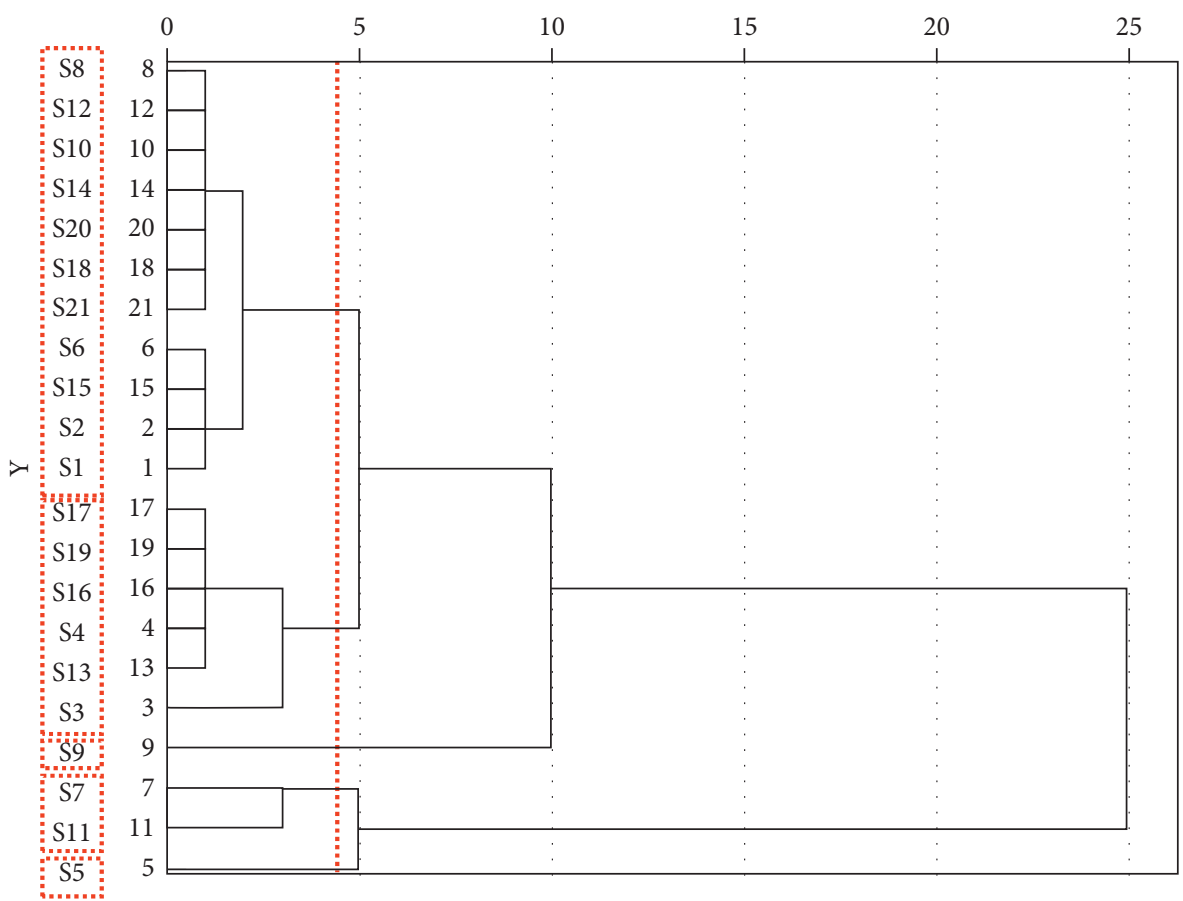

FIgURE 4: Clustering analysis graph of the 21 licorice samples.

compounds of 21 batches in licorice. Combined with the similarity of HPLC fingerprints, the contents of licorice from different sources show considerable differences. Although the contents of liquiritin and glycyrrhizic acid in the 21 batches of licorice were determined and accorded with the 2020 edition of the Chinese Pharmacopoeia, the licorice content of different sources is different. Furthermore, it is hard to comprehensively control the quality of licorice by using only two detection indicators. In previous studies, the contents of 4 compounds were determined by HPLC for the quality control of licorice [32]. In this study, considering the shortcomings of the multicomponents' determination, we have combined characteristic fingerprint with QAMS to improve the quality control and evaluation of licorice. We first used QAMS to simultaneously determine the content of 11 compounds from licorice, and the feasibility of the method was verified. In addition, the contents' variations of 11 compounds between QAMS and ESM are within the range of $5 \%$, which indicated that there is no significant difference between the two methods. Therefore, the HPLC-QAMS method could be used for replacement of ESM in case of lack of reference substance. And it is an innovative method to provide for the comprehensive quality control of licorice.

Hierarchical cluster analysis is a chemical pattern recognition and classification evaluation method, which is used to find relatively isomorphic cluster objects based on measurement characteristics. The most similar samples are clustered preferentially [28]. In order to obtain the HCA dendrogram, the 11 components were set as variables, and Ward's method was selected as the cluster method and squared Euclidean distance as the interval measurement [33]. The results showed that citrus and grape were divided into five main groups, and the content of licorice is significantly different in the same area.
The factors included soil type, climate, humidity, and temperature, harvesting time, planting patterns, and dying methods, and other factors influenced the content of licorice. Therefore, the quality control of licorice should be taken seriously by researchers.

\section{Conclusions}

The quality evaluation method of the eleven components in 21 batches of licorice was established using QAMS methods, combined with HPLC fingerprints analyses and HCA method. The contents of the eleven components in licorice samples were simultaneously determined by the QAMS method. Glycyrrhizic acid was selected as the internal reference substance. In order to compare the consistency of QAMS and EMS, the angle cosine value and the correlation coefficient were calculated, and the results indicated that no significant difference existed between the newly established QAMS method and the traditional ESM.

Licorice has traditional efficacy of strengthening the spleen and benefiting vital qi and heat-clearing and detoxifying. The eleven compounds have been reported to be correlated with the traditional efficacy of licorice. So, eleven components were determined simultaneously in this experiment by QAMS-HPLC, which reflected more information than the one component (glycyrrhizic acid) determined in pharmacopoeia. Meanwhile, only one single reference substance was used in this experiment, which greatly reduced the cost of testing and was easily used for the scientific research and supervised application. On the basis of these results, the quality of licorice could be visible and better identified comprehensively by HCA and similarity analysis. Therefore, 
QAMS method combined with the HPLC fingerprint might provide an overall chemical characteristic, along with similarity analysis and HCA of synthesis. And this effective method has promising wide applications in the quality control of licorice and medicinal plants.

\section{Data Availability}

The data used to support the findings of this study are available from the corresponding author upon request.

\section{Conflicts of Interest}

The authors declare no conflicts of interest.

\section{Authors' Contributions}

Tingting Li, Hao Zhou, and Jiahua Ma contributed equally to this work. All the authors developed and studied the manuscript. Xueyan Fu designed this article. Tingting Li and Hao Zhou conducted the literature survey and drafted the paper. Jiahua Ma oversaw the work and revised the manuscript.

\section{Acknowledgments}

This work was supported by the Ningxia Natural Science Foundation Project (Grant no. 2018AAC03097), the 12th Five-Year National Science and Technology Support Project (Grant no. 2013BAI05B01), the National Nature Science Foundation of China (Grant nos. 81860708 and 81560647), Ningxia Key Research and Development Program (2018BEG03016), the Undergraduate Innovation and Entrepreneurship Training Program of Ningxia Medical University in 2019(19), the Ningxia Higher Education School First-Class Discipline Construction Project (Chinese Medicine) Funded Project (Grant no. NXYLXK2017A06), and School Level Project of Ningxia Medical University (XM2020022). The authors would like to thank Dr. Dong Lin and Fang Xu from Ningxia Medical University for correcting the English grammar of this article.

\section{References}

[1] S. Hongxiang and P. HangjJun, "Immunological adjuvant effect of Glycyrrhiza uralensis saponins on the immune responses to ovalbumin in mice," Vaccine, vol. 24, pp. 19141920, 2006.

[2] C. Yu, Y. Zhang, K.-X. Gao et al., "Serotonergically dependent antihyperalgesic and antiallodynic effects of isoliquiritin in a mouse model of neuropathic pain," European Journal of Pharmacology, vol. 881, p. 173184, Article ID 173184, 2020.

[3] Y.-C. Wang and Y.-S. Yang, "Simultaneous quantification of flavonoids and triterpenoids in licorice using HPLC," Journal of Chromatography B, vol. 850, no. 1-2, pp. 392-399, 2007.

[4] N. A. Mamedov and D. Egamberdieva, "Phytochemical constituents and pharmacological effects of licorice: a review," Plant and Human Health, vol. 3, pp. 1-21, 2019.

[5] R. S. Kimyon, Y. L. Liou, J. P. Schlarbaum, and E. M. Warshaw, "Allergic contact dermatitis to licorice root extract,” Dermatitis, vol. 30, no. 3, pp. 227-228, 2019.
[6] European Pharmacopoeia Commission, European Pharmacopoeia 9.0, pp. 1416-1417, European Directorate for the Quality of Medicines and HealthCare, Strasbourg, France, 2016.

[7] The United States Pharmacopieial Convention, Pharmacopeia 42, The United States Pharmacopieial Convention: America, Rockville, MD, USA, 2018.

[8] Editorial Board of Japan Pharmaceutical Administration, Japanese Pharmacopoeia, Japan Health Ministry Press, Tokyo, Japan, 2016.

[9] Chinese Pharmacopoeia Commission, Pharmacopoeia of the People's Republic of China, Part 1, China Medical Science, Beijing, China, 2020.

[10] C. Anzhen, S. Lei, Y. Hang, W. Aiying, L. Jingguang, and M. Shuangcheng, "Simultaneous qualitative and quantitative analysis of 11 active compounds in rhubarb using two reference substances by UHPLC," Journal of Separation Science, vol. 41, pp. 3686-3696, 2018.

[11] X. Liu, Q. Li, C. Lv et al., "Combination of the advantages of chromatographic methods based on active components for the quality evaluation of licorice," Journal of Separation Science, vol. 38, no. 24, pp. 4180-4186, 2015.

[12] M. Kimura, H. Inoue, K. Hirabayashi, H. Natsume, and M. Ogihara, "Glycyrrhizin and some analogues induce growth of primary cultured adult rat hepatocytes via epidermal growth factor receptors," European Journal of Pharmacology, vol. 431, no. 2, pp. 151-161, 2001.

[13] Y. Liu, X. Xu, R. Xu, and S. Zhang, "Renoprotective effects of isoliquiritin against cationic bovine serum albumin-induced membranous glomerulonephritis in experimental rat model through its anti-oxidative and anti-inflammatory properties," Drug Design, Development and Therapy, vol. 13, pp. 37353751, 2019.

[14] Z.-Y. Wu, L.-X. Sang, and B. Chang, "Isoflavones and inflammatory bowel disease," World Journal of Clinical Cases, vol. 8, no. 11, pp. 2081-2091, 2020.

[15] P. Wu, T. Yu, J. Wu, and J. Chen, "Licochalcone a induces ROS-mediated apoptosis through TrxR1 inactivation in colorectal cancer cells," BioMed Research International, vol. 2020, p. 1, Article ID 5875074, 2020.

[16] B. Goel, A. Sharma, N. Tripathi et al., "In-vitro antitumor activity of compounds from Glycyrrhiza glabra against C6 glioma cancer cells: identification of natural lead for further evaluation," Natural Product Research, vol. 34, pp. 1-4, 2020.

[17] Y.-B. Zhang, J. DA, J.-X. Zhang et al., "A feasible, economical, and accurate analytical method for simultaneous determination of six alkaloid markers in Aconiti Lateralis Radix Praeparata from different manufacturing sources and processing ways," Chinese Journal of Natural Medicines, vol. 15, no. 4, pp. 301-309, 2017.

[18] Y. Li, Y. Zhang, Z. Zhang, Y. Hu, X. Cui, and Y. Xiong, "Quality evaluation of gastrodia elata tubers based on HPLC fingerprint analyses and quantitative analysis of multicomponents by single marker," Molecules, vol. 24, no. 8, p. 1521, 2019.

[19] S. Wang, Y. Xu, Y. Wang et al., "Simultaneous determination of six active components in oviductus ranae via quantitative analysis of multicomponents by single marker," Journal of Analytical Methods in Chemistry, vol. 2017, p. 1, Article ID 9194847, 2017.

[20] Y. Chen, S.-B. Zhu, M.-Y. Xie et al., "Quality control and original discrimination of Ganoderma lucidum based on high-performance liquid chromatographic fingerprints and combined chemometrics methods," Analytica Chimica Acta, vol. 623, no. 2, pp. 146-156, 2008. 
[21] X.-Y. Gao, Y. Jiang, J.-Q. Lu, and P.-F. Tu, "One single standard substance for the determination of multiple anthraquinone derivatives in rhubarb using high-performance liquid chromatography-diode array detection," Journal of Chromatography A, vol. 1216, no. 11, pp. 2118-2123, 2009.

[22] B. Su, "Recent progress on fingerprint visualization and analysis by imaging ridge residue components," Analytical and Bioanalytical Chemistry, vol. 408, no. 11, pp. 2781-2791, 2016.

[23] L. D. Wei, Z. Ming, S. Y. Dong, S. Zhe, W. C. Chen, and Y. W. Dong, "Determination and quality evaluation of green tea extracts through qualitative and quantitative analysis of multi-components by single marker (QAMS)," Food Chem, vol. 197, pp. 1112-1120, 2016.

[24] Z. R. Yi, Z. J. Hua, and W. M. Tian, "Studies on the saponins from the root of Glycyrrhiza uralensis Fisch," Yao Xue Xue Bao, vol. 21, pp. 510-515, 1986.

[25] A. S. Ammosov and V. I. Litvinenko, "Triterpenoids of plants of Glycyrrhiza L. and Meristotropis Fisch. et Mey Genuses (a review)," Pharmaceutical Chemistry Journal, vol. 37, no. 2, pp. 83-94, 2003.

[26] Q. L. Sen, X. Ying, D. W. Long et al., "Ligand fishing with functionalized magnetic nanoparticles coupled with mass spectrometry for herbal medicine analysis," Analytical Bioanalytical Chemistry, vol. 399, pp. 1223-1231, 2011.

[27] Y. D. Mei, C. Y. Xu, K. L. Yuan, and G. X. Mei, "Quality evaluation and regional analysis of psoraleae fructus by HPLC-DAD-MS/MS plus chemometrics," Chinese Herbal Medicines, vol. 2, pp. 216-223, 2010.

[28] K. P. Raj, L. Seockheon, K. S. Raj, and K. S. Pratap, "Chemometric application in classification and assessment of monitoring locations of an urban river system," Analytica Chimica Acta, vol. 582, pp. 390-399, 2007.

[29] C.-Q. Wang, X.-H. Jia, S. Zhu, K. Komatsu, X. Wang, and S.-Q. Cai, "A systematic study on the influencing parameters and improvement of quantitative analysis of multi-component with single marker method using notoginseng as research subject," Talanta, vol. 134, pp. 587-595, 2015.

[30] X. Ma, L. Fan, F. Mao et al., "Discrimination of three Ephedra species and their geographical origins based on multi-element fingerprinting by inductively coupled plasma mass spectrometry," Scientific Reports, vol. 8, no. 1, p. 10271, 2018.

[31] Y. Rui, L. W. Dong, Y. B. Chuan et al., "Simultaneous determination of $18 \alpha$-glycyrrhizic acid and $18 \beta$-glycyrrhizic acid in three licorice samples from different origin by HPLC," Chinese Journal of Pharmaceutical Analysis, vol. 36, no. 6, pp. 1065-1071, 2016.

[32] Y. Rui, Y. B. Chuan, Y. S. Ma et al., "Simultaneous determination of liquiritin,isoliquiritin,liquiritigenin and isoliquiritigenin in Glycyrrhiza uralensis Fisch.,Glycyrrhiza glabra L., and Glycyrrhiza inflata Bat. by HPLC," Chinese Journal of Pharmaceutical Analysis, vol. 10, pp. 1729-1736, 2016.

[33] X. Yan, W. Wang, Z. Chen et al., "Quality assessment and differentiation of Aucklandiae Radix and Vladimiriae Radix based on GC-MS fingerprint and chemometrics analysis: basis for clinical application," Analytical and Bioanalytical Chemistry, vol. 412, no. 7, pp. 1535-1549, 2020. 